\title{
Introduction Des Organismes Génétiquement Modifiés(OGM) Dans L'agriculture En Côte d'Ivoire: Etat De Connaissance Et Souhaits Des Populations De Six (6) Localités Du Sud Du Pays
}

\author{
Boraud N'takpé Kama Maxime \\ Université de Cocody-Abidjan, UFR Bioscience \\ Laboratoire de Botanique, Abidjan \\ Ake Séverin \\ Université de Cocody-Abidjan, UFR Bioscience \\ Laboratoire de Physiologie Végétale, Abidjan \\ Jacques Gasquez \\ Institut National de Recherche Agronomique, Dijon, .France
}

doi: 10.19044/esj.2016.v12n30p112 URL:http://dx.doi.org/10.19044/esj.2016.v12n30p112

\begin{abstract}
Transgenic plants with their huge potential as the high yields, resistance to diseases, insects and water stress seem to be a solution for Africa and could contribute to poverty reduction and the increase of agricultural production in this area of the world often face starvation. However GMOs dragging a trail of controversy and risk maligned with a series of impacts that concern the human health, environmental, social and economic. Is the dangerousness of transgenic plants a concern people of Ivory Coast? Investigations were carried out on 400 farmers and 1000 educated people in through a series of questions.
\end{abstract}

Keywords: Transgenic plant, farmer, intellectual, Ivory Coast

\section{Introduction}

L'Afrique sub-saharienne reste la partie du globe où le nombre de « sous-alimentés » atteindra 205 millions d'habitants en 2015 (FAO, 2005). Dans cette région du monde, la production céréalière annuelle par habitant est la plus faible au monde avec $128 \mathrm{~kg}$, pour des rendements à l'hectare inférieurs à une tonne (FAO, 2002). Globalement, l’Afrique reste dépendante de l'assistance internationale et a reçu, en 2000, quelques 2,8 millions de tonnes d'aide alimentaire, soit plus du quart du volume mondial total (Saito, 2000). 
En 2008, la crise céréalière survenue par la hausse des prix des denrées alimentaires a fortement perturbé les économies africaines et entrainer des troubles à l'ordre public, semblables à des guerres civiles dans presque toutes les grandes villes africaines (www.monde-solidarite.org du 18/10/2008).

Face à toutes ces difficultés, les plantes transgéniques avec leurs énormes potentialités (rendements élevés, résistances aux maladies, aux insectes et au stress hydrique), (Jaglo-Ottosen et al., 1998) semblent représenter une solution pour l'Afrique et pourraient contribuer à la réduction de la pauvreté et à l'augmentation de la production agricole dans cette zone du monde.

La production du riz en Asie a augmenté de 170 \% depuis 1962, alors que la population passait de 1,6 à 3,7 milliards. L'augmentation de la production a exigé moins de $30 \%$ des terres en plus, grâce à de nouvelles variétés de riz obtenues par des efforts d'hybridation et qui ont permis l'introduction de nouvelles caractéristiques telles que les gènes de nanification et ceux de la qualité des grains (Hei et al., 2002).

Flowers et al. (2000) ont mis au point une variété de riz tolérant la salinité par l'ajout d'un gène. Un riz basmati transgénique résistant à la sécheresse et à l'eau saumâtre (Morin, 2002). Des gènes de levure favorisent son développement dans de tels milieux. Les anglo-saxons les appellent « plantes reviviscentes ». Elles sont capables de résister à des sécheresses intenses et de renaître à la moindre goutte de pluie. Elles survivent alors que leur teneur en eau ne représente que $5 \%$ de leur poids, deux fois moins que dans le riz par exemple. Ce qui distingue ces végétaux, c'est leur concentration élevée en tréhalose, un sucre que l'on trouve aussi chez les bactéries, les champignons et les invertébrés et qui semble jouer un rôle dans la résistance au stress hydrique.

Avec l'utilisation des cotons Bt, des pays comme l'Inde et la Chine ont nettement amélioré leur production avec respectivement 580 et 1043 $\mathrm{kg} / \mathrm{ha}$. Tout ceci accompagné d'une réduction drastique de l'utilisation des pesticides (www.libertyindia.org/policy reports/cotton info march 2002.htm).

La question des impacts potentiels des OGM sur l'environnement a donné lieu à une abondante littérature. Le développement du soja RoundupReady (soja RR) a largement évincé les autres herbicides employés naguère au profit du glyphosate. Ainsi, dans la quinzaine d'états plus grands producteurs de soja, entre 1995 et 2000, la part de culture de soja recevant du glyphosate est passée de $20 \%$ à $62 \%$ tandis que celle traitée à l'Imazéthapyr a chuté de 44 à $12 \%$; celle traitée à la pendimethaline a baissé de 26 à $11 \%$, à la trifluraline de 20 à 14 \% (Doré et Varoquaux, 2006).

Il faut aussi considérer les retombées à plus long terme de l'emploi de glyphosate et des plantes transgéniques tolérantes. La consommation de 
glyphosate augmente rapidement avec la diffusion des plantes transgéniques tolérantes, mais aussi avec le développement des techniques de cultures simplifiées (Woodburn, 2000). En 1999-2000, le glyphosate était répandu sur environ $70 \%$ des surfaces cultivées avec les plantes transgéniques tolérantes (Verfaillie, 2001).

L'évaluation des OGM dans les exploitations pose divers problèmes méthodologiques, souvent peu pris en compte dans les controverses sur les résultats, mais analysés assez finement par plusieurs agro économistes qui s’y sont essayés (Heimlich et al., 2000, Lemarié, 2000, Mara, 2001 ; Lin et al., 2001).

Cependant, les organismes génétiquement modifiés traînent un cortège de polémiques et de risques décriés qui concerneraient la santé humaine, l'environnement, les risques économiques et sociaux liés aux questions de brevetage du vivant, au problème d'appropriation des ressources génétiques locales et des connaissances traditionnelles par les firmes multinationales (Oury, 2006)

Face aux applications potentielles des biotechnologies, les attitudes apparaissent divergentes et teintées d'émotivité (Lepoivre, 2002). Les techniques de transformations génétiques provoquent, chez certains groupes, des réactions extrêmement prudentes, au point de proposer des moratoires sur toute action, y compris l'expérimentation, alors que ces techniques paraissent aptes à résoudre des problèmes agronomiques importants (Lepoivre, 2002).

A l'autre extrémité de la palette des réactions, des scientifiques et industriels entretiennent un engouement sans réserve fondé sur les réalisations scientifiques et les perspectives d'application que relèvent quotidiennement la presse scientifique.

En Côte d'Ivoire, il existe la charte de la coalition ivoirienne de biovigilance (CI-BIOV). Cette organisation a pour missions :

- de participer au processus d'élaboration des lois d'application ;

- d'analyser et faire des propositions sur les projets de lois concernant les OGM ;

- $\quad$ d'informer et de sensibiliser le public sur les OGM, leurs enjeux et toute introduction de produits OGM en Côte d'Ivoire (www.inadesfo.net).

Selon cette organisation, la législation sur les organismes génétiquement modifiés est prête et a été soumise à l'Assemblée Nationale pour adoption. En attente de l'adoption de cette loi, aucun organisme génétiquement modifié ne circule officiellement dans le pays. Toutes ces divergences citées plus haut sur les OGM laissent les autorités dans la difficulté à adopter une position définitive (Boraud, 2010).

Nous avons donc initié cette étude dans le seul objectif d'avoir l'état de connaissance et les souhaits de quelques populations ivoiriennes sur les 
OGM. Il s’agissait de savoir si les nationaux, dans leur majorité, savent ce que c'est que les organismes génétiquement modifiés. S’ils les connaissent, quels usages veulent-ils en faire ? Pour répondre à ces questions, une série d'enquêtes au sein d’un échantillon de la population a été initiée.

Nos résultats devraient permettre aux populations d'avoir une opinion objective sur les organismes génétiquement modifiés et aux décideurs de posséder des éléments de base de décisions.

\section{Matériel et méthode}

\section{Populations cibles des enquêtes}

Deux fiches ont été élaborées en fonction de la qualité de l'individu interrogé (Annexes 1 et 2). Dès le début de l'enquête, deux catégories de personnes ont été ciblées.

Il s'agit d'une part des intellectuels considérés comme des personnes ayant obtenu le baccalauréat et qui ont fait des études supérieures. Dans cette catégorie ont été répertoriés les étudiants inscrits à tous les nivaux de l'U.F.R. Biosciences car disponibles et accessibles, les chercheurs du CNRA, les enseignants chercheurs de l'Université de Cocody (toutes les disciplines confondues) et les responsables du Ministère de la Recherche Scientifique.

D'autre part, les paysans de tous niveaux d'étude qui travaillent au quotidien la terre et qui ont la réalité du terrain.

\section{Enquêtes réalisées}

400 paysans et 1000 intellectuels ont étés interrogés à travers une série de questionnaires disponibles sur deux fiches d'enquêtes (annexes 1 et 2).

Concernant les paysans, l'enquête a été réalisée entre Mai et Décembre 2006 à travers les localités d'Agboville, de Rubino, d'Adzopé, de Gagnoa et d'Aboisso à cause de leur proximité d'Abidjan et surtout pour leur niveau d'expression en français. A chaque rencontre, les paysans ont été aidés dans le remplissage du formulaire par un guide local et les réponses ont été transcrites sur les fiches d'enquêtes par nous-mêmes.

En ce qui concerne les responsables du Ministère de la Recherche Scientifique, les enseignants-chercheurs et les étudiants, les formulaires de questions ont étés distribués et colletés après une ou deux semaines. Cette enquête s'est déroulée entre Septembre 2005 et Juin 2006.

L'analyse a consisté à faire les statistiques pour évaluer l'effectif des paysans et d'intellectuels qui connaissent ou ont entendu parler des OGM, leur source d'information, le type de culture transgénique éventuelle qu'ils souhaitent avoir, le type de loi souhaitée et les caractères agronomiques que les ivoiriens désir que les cultures acquièrent. 


\section{Résultats}

\section{Résultats d'enquêtes chez les paysans}

Sur la connaissance des OGM et par rapport à la source d'information

A la question «connaissez-vous les OGM? », l'enquête menée auprès de 400 paysans des zones rurales d'Azaguié, d'Adzopé, d'Aboisso, d’Agboville, de Rubino et de Gagnoa montre que 263 individus, soit 65,72 \% des paysans ignorent l'existence des organismes génétiquement modifiés contre 137 (34, 28 \%) qui en connaissent l'existence.

Concernant les 137 paysans qui connaissent ou ont entendu parler des OGM, à la question de savoir « quelle est votre source d'information »?, la grande majorité, soit 121 (88,32 \%) des personnes ont avancé que l'information leur a été donnée par des interlocuteurs occasionnels et 16 (11,68 \%) en ont entendu parler à la télévision lors des journaux télévisés.

\section{Avis des paysans}

A la question «Êtes-vous pour ou contre la culture des plantes transgéniques en Côte d'Ivoire ? », sur les 137 paysans qui ont su de quoi il s’agissait, 35 (25,55 \%) ont été pour une culture des plantes transgéniques en Côte d'Ivoire, tandis que 27 (19,7\%) des paysans ont été contre, 75 (54,74 \%) des paysans n’ont pas eu d'opinion et ont déclaré s'en remettre aux structures d'encadrement comme l'ANADER (Agence Nationale d'Appui au Développement Rural) qui, selon eux, sont capables de leur donner la réponse correcte. Quand aux 27 paysans qui sont contre l'introduction des cultures transgeniques, leurs inquiétudes ont été que les OGM provoqueraient des problèmes de santé et un risque pour la pollution de l'environnement.

\section{Raisons évoquées pour l'acceptation des OGM}

A la question «pourquoi souhaitez-vous avoir des plantes transgéniques ? », les paysans favorables à l’introduction de cultures transgéniques ont répondu unanimement que de telles cultures leur procureraient une augmentation substantielle des rendements, donc naturellement l'augmentation de leurs revenus.

\section{Types de plantes transgéniques souhaitées}

Lorsqu'on pose la question, «Quelles types de plantes transgéniques aimeriez-vous avoir ? », sur les 35 paysans qui ont été favorables, 15 (42,85 \%) ont été pour uniquement les cultures céréalières et 20 (57,15\%) ont été pour les cultures de rentes surtout le caféier et le cacaoyer. 


\section{Caractères agronomiques souhaitées pour les plantes génétiquement modifiées}

À la question «Quel caractère aimeriez-vous que la plante transgénique acquiert? », tous les 35 paysans (100 \%) de façon unanime ont été pour avoir, en premier, des plantes qui résistent au stress hydrique; suivent celles qui ont un rendement élevé et enfin celles qui résistent à un herbicide et à un insecticide à la fois.

Comment ils souhaiteraient faire la succession culturale avec l'éventuelle introduction de cultures transgéniques, surtout, celles résistantes aux herbicides?

Cette question a semblé bien intéressante car selon certains paysans, elle pourrait aider à résoudre les problèmes de pressions démographiques sur les terres. En général, pour les cultures vivrières annuelles, l’on est obligé de défricher de nouvelles terres à cause des problèmes d'enherbement. L'introduction d'une culture résistante à un herbicide total leur permettrait d'exploiter pendant plusieurs années la même parcelle et pourrait résoudre également le problème de déforestation et les conflits fonciers permanents liés à la défriche de nouvelles parcelles.

Les paysans ont proposé dans la succession culturale, d’abord une culture vivrière, suivie d'une culture pérenne. Mais, en même temps, ils ont proposé compte tenu du coût élevé des intrants agricoles que les scientifiques leur offrent des plantes transgéniques pour la culture.

\section{Résultats d'enquêtes chez les intellectuels Sur la connaissance des OGM}

A la question « connaissez-vous les OGM ? », nous avons recueilli 971 réponses affirmatives contre 29 réponses négatives. Cela représente 97,10 \% des individus interrogés qui sont informés de l'existence des organismes génétiquement modifiés.

\section{Par rapport à la source d'information}

La plupart des individus interrogés ont plusieurs sources d’informations dont la plus importante est la télévision et la radio (800). Ensuite, viennent les journaux et les interlocuteurs (550). Les livres et l'internet (300) donnent l'information sensiblement au même pourcentage.

\section{Sur la prise de position pour ou contre les plantes transgéniques et les raisons}

A la réponse à cette question, 571 individus ont été contre les OGM soit 58,80 \% de rejet des cultures transgéniques par la population intellectuelle et 400 (41,20\%) des individus ont été pour. Les raisons majeures du rejet ont été les problèmes de santé (430 individus) et la 
pollution de l'environnement par les flux de gènes des cultures transgéniques (200 individus) qui transmettraient leurs résistances aux espèces voisines sauvages. Ces espèces sauvages, une fois en possession du transgène deviendront plus vigoureuses, prendront un avantage certain sur les espèces non transgéniques et se répandront partout réduisant la biodiversité végétale. D’autres raisons évoquées par les opposants aux cultures transgéniques concerneraient surtout 'un problème d'éthique'. Ils conçoivent assez difficilement que l'on transfère un gène animal à un végétal, alors que ce n’est pas le cas pour les cultures traditionnelles. Selon eux, la science irait au delà des principes divins.

La raison essentielle avancée par ceux qui sont favorables aux plantes transgéniques est d'ordre économique. Selon cette catégorie, les plantes transgéniques pourraient améliorer la qualité des cultures et assurer des rendements plus élevés et cela permettra aux paysans de vendre plus et de gagner un peu plus d'argent.

\section{Caractéristiques souhaitées pour les plantes transgéniques}

Concernant les 400 individus qui ont été favorables, les plantes transgéniques devraient avoir en priorité les caractéristiques suivantes: d'abord, les plantes doivent être résistantes aux herbicides (232 individus) ; ensuite, avoir une résistance au stress hydrique (80 individus), un rendement élevé (44 individus) et être résistantes aux maladies (44 individus).

\section{Types de plantes transgéniques souhaitées}

A la question «Quel type de plante souhaitez-vous voir génétiquement modifiée? », 244 individus ont été favorables aux cultures céréalières comme le riz, le mil et le maïs, 112 pour les cultures vivrières comme la banane plantain, l'aubergine et les piments, 44 pour les cultures de rente et 130 pour tous les autres types de cultures.

\section{Types de législations souhaités}

A la question « Êtes-vous pour une législation? », 771 individus soit (77,10 \%) sont favorables à une législation sur les OGM contre 200 (20\%) individus.

Quand à la loi modèle dont il faut s’inspirer pour avoir une législation sur les OGM en Côte d’ivoire, 346 individus soit 44,87 \% ont été pour une législation modèle s’inspirant de celle de l'Union Européenne (opposants), 232 individus ont été favorables à la loi modèle des USA (favorables), soit 30,10 \% et 193 individus, soit 25, 03 \% ont été favorables à la loi modèle proposée par l’Union Africaine.

En ce qui concerne les précautions à prendre avant l'introduction des OGM en Côte d'ivoire, 614 individus, soit 79,63 \% des intellectuels ont 
souhaité que le pays se dote de structures adéquates pour exiger et suivre la traçabilité des produits OGM qui seront sur le territoire ivoirien.

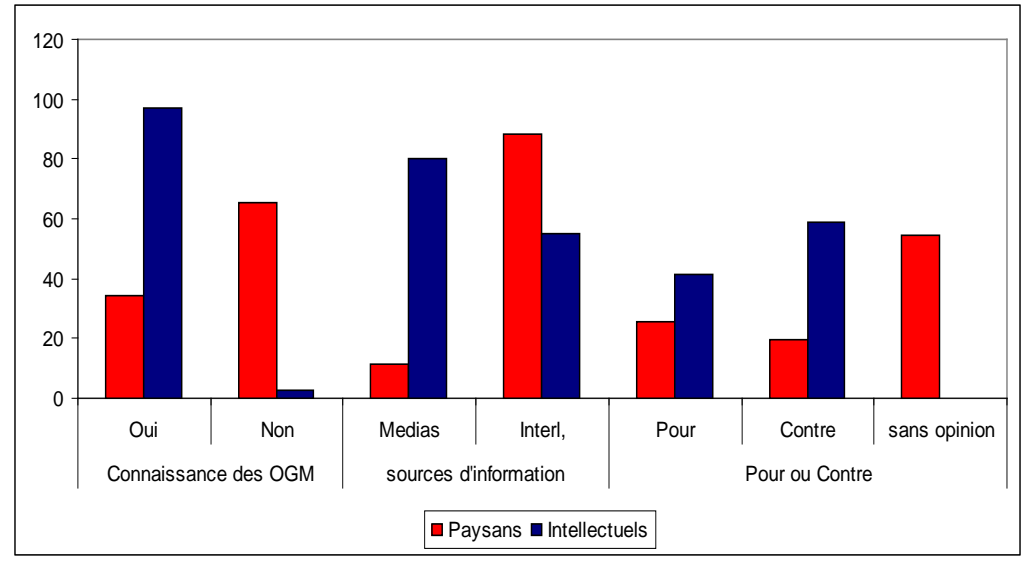

Figure 1 : connaissance, source d'information et avis de quelques populations ivoiriennes sur les OGM

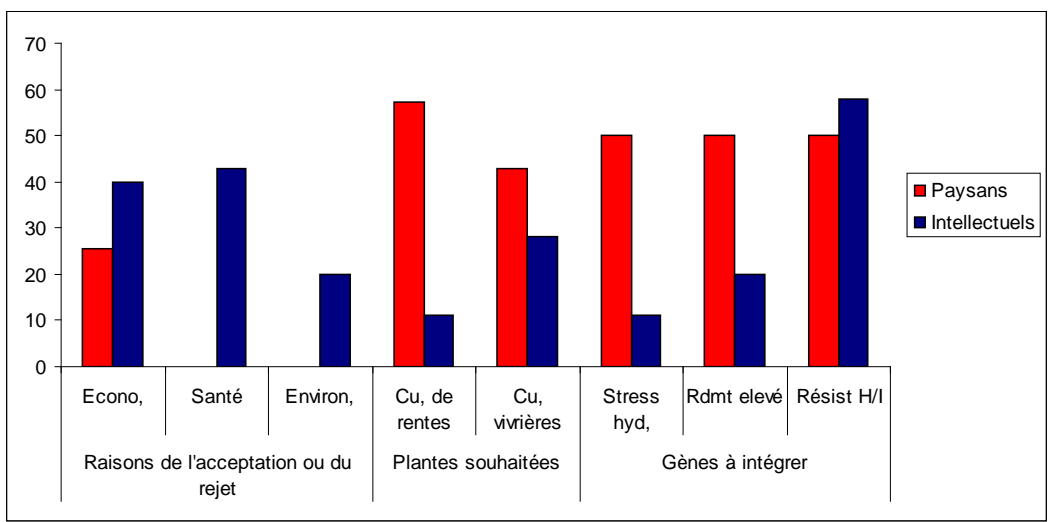

Figure 2 : Causes du rejet où de l'acceptation, plantes transgéniques souhaitées et types de caractères chez quelques populations ivoiriennes

\section{Discussion}

Deux groupes d’ivoiriens sont interrogés dans notre étude, les intellectuels d'une part et le monde paysan d'autre part.

En ce qui concerne le monde paysan, les localités visitées ont un fort taux de scolarisation et la plupart des paysans rencontrés se sont exprimés plus ou moins bien en français. Les villages sont dans l'ensemble électrifiés et les moyens de communications modernes tels que la télévision, la radio et le téléphone existent à ces endroits. Malgré tous ces moyens de communications, 65,72 \% des paysans interrogés ont ignoré l'existence ou n’ont jamais entendu parler des OGM.

Si nous projetons ces données à l'ensemble du pays et compte tenu des moyens de communications inexistants dans les zones reculées, 80 à 90 
\% des paysans et des populations des zones rurales pourrait n’avoir jamais eu à entendre parler des OGM.

Les 8,75 \% de paysans favorables à l'introduction de cultures OGM sont des agriculteurs possédant, soit à la fois de vastes plantations de caféiers, de cacaoyers, d’hévéa, d'ananas etc.., soit une vaste plantation d'une des cultures citées.

Concernant les intellectuels, 97,1 \% interrogés connaissent l'existence des organismes génétiquement modifiés. Ce taux très élevé est lié à la qualité de l'échantillonnage. En effet, la majorité de l'effectif de l'enquête a été recrutée dans le monde universitaire, relativement informé sur les évènements de la planète. La source de l'information est dominée par la télé et la radio (totalité des individus connaissant les OGM), suivie des journaux (550 individus) et par divers interlocuteurs (400 individus). Toutes les personnes interrogées n’ont jamais eu à manipuler ou à suivre de très près un évènement concernant les OGM.

Malgré cela, 40 \% ont été favorables aux plantes transgéniques contre $57,1 \%$.

Tous ceux qui ont été favorables aux plantes transgéniques, que ce soit les paysans ou les intellectuels, ont désiré avoir des cultures avec des rendements élevés.

Les intellectuels ont été plutôt favorables à une loi sur les OGM s’inspirant du modèle de l'Union européenne.

En Europe, dans une enquête Eurobaromètre 55,2 (Gallais et al., 2006), intitulée « les Européens, la science et la technologie », réalisée en décembre 2001 à la demande de la direction générale de la recherche de la commission européenne, et à la question « êtes-vous d'accord ou pas avec les aliments transgéniques » « je ne veux pas ce type d’aliment », $71 \%$ des européens ont répondu ne pas en vouloir et seulement $17 \%$ en voulait bien (Bonny, 2003). Dans cette enquête qui a porté sur 16029 personnes (en moyenne 1000 par état membre), la variation entre pays a été bien plus forte que la variation selon les critères socio- démographiques comme l’âge, le sexe, le niveau d'instruction, la profession, etc. Ainsi, la Grèce est le pays le plus opposé avec $93 \%$ des personnes interrogées et les Pays-Bas sont le pays le moins hostile avec seulement 52 \% d’opposition. La France avec 79 \% des personnes interrogées opposées aux aliments transgéniques, figure parmi les pays les plus hostiles, juste derrière la Grèce. Il n’y a pas de déterminants géographiques manifestes dans l'opposition aux aliments issus de plantes génétiquement modifiées, même si l'on peut regrouper quelques pays comme la Finlande et la Suède avec 77 \% d’opposition et la Grande Bretagne, L'Irlande, Les Pays-Bas et la Belgique avec en moyenne 60 \% d'opposition.

Cette grande variation entre pays et la plus faible variation entre catégories sociodémographiques tend à montrer un fort effet de la vie sociale 
et politique à l'intérieur de chaque pays sur la position des consommateurs. Comme le soulignent De Cheveigné et al., (2002), cette variation est liée à la différence culturelle mais aussi au niveau des différences dans les débats publics, l’intervention des gouvernants et le développement économique.

Malgré le peu de débat sur les OGM dans la société ivoirienne, seul $57,1 \%$ des intellectuels s’opposent aux plantes transgéniques. Ce qui est largement en deçà des seuils européens.

Au niveau mondial, une enquête « Environics International » réalisée en 2000 (Le Déaut, 2005) montrait que 72 \% des Chinois, 69 \% des Indiens et $66 \%$ des Américains considèrent que les avantages des biotechnologies sont plus importants que les risques, alors que ces chiffres étaient de $39 \%$ pour les Espagnols, $34 \%$ pour les Italiens et seulement 22 \% pour les Français. Dans cette enquête, il apparaît le comportement particulier des Pays-Bas par rapport aux autres pays européens, avec un taux de $55 \%$.

Dans cette même enquête Eurobaromètre, 54,6 \% des Européens interrogés considéraient les aliments contenant des OGM comme dangereux pour leur santé (avec toutefois 25,6 \% sans opinion) et 59,4 \% répondaient que les plantes transgéniques auraient des effets négatifs sur l'environnement. Cela peut donc traduire un rejet des plantes transgéniques par suite de crainte sur la santé et l'environnement. Cependant, le rejet des plantes transgéniques en Europe apparaît lié au rejet des biotechnologies dans leur ensemble.

En Côte d'Ivoire, par contre, le rejet des plantes transgéniques est plus lié à la peur de ce qu’on ne sait pas qu'au rejet de la biotechnologie comme en Europe. Les ivoiriens, dans leur ensemble, ont peur des risques environnementaux.

L’absence de débats au sein de toute la société ivoirienne sur les OGM, emmène les médias francophones, surtout français à majorité antiOGM à donner seuls l'information donc à orienter l'information selon leurs propres convictions.

Il est important de rappeler que le traitement de l'information concernant les OGM est alarmant.

Pour preuve, voilà des faits récents. Selon le journal français le monde « Lancée le 23 avril 2004, par le Crii-Gen, une association écologiste (présidée par l'ancien ministre français de l'Environnement Corinne Lepage), l'«affaire» du maïs Mon 863 a fait grand bruit. Cet organisme génétiquement modifié, commercialisé par le géant américain Monsanto, induirait de graves malformations rénales chez le rat. De quoi légitimement faire craindre de sérieux risques pour la santé humaine en dépit des avis rassurants donnés, jusque-là, par la communauté scientifique sur la dangerosité des OGM. «Pour la première fois, on découvre que les experts admettent que l'ingestion d'OGM a des effets significatifs sur les animaux», déclare ce jour-là Corinne 
Lepage, dans une interview au Monde, en appui d'un article intitulé «l'expertise confidentielle sur un inquiétant maïs transgénique».

Le Crii-Gen vient en effet de se procurer, non sans mal, auprès de la Commission du génie biomoléculaire (CGB), l'une des instances chargée avec l'Afssa (Agence française de sécurité sanitaire des aliments) d'instruire les demandes d'autorisation d'OGM pour le compte du gouvernement français, les résultats détaillés d'une étude de toxicité effectuée sur des rats nourris pendant 90 jours avec du Mon 863 comparés à des rats témoins alimentés avec du maïs conventionnel.

Dans son avis daté du 28 octobre 2003, la CGB constate «des différences statistiques significatives» sur des indicateurs clés comme «l'hématologie (globules blancs, lymphocytes), la biochimie clinique (glycémie) ainsi que le poids des reins» et estime ne pas être «en mesure de conclure à l'absence de risque pour la santé animale».

Mais, contrairement à ce que laisse entendre Corinne Lepage, les scientifiques sont loin d'être unanimes. Dans un avis publié le 6 novembre 2003, l'Afssa considère que les différences constatées sur les rats soumis à un régime transgénique "sont sans signification biologique» et conclut que «la consommation» du maïs Mon 863, qui a la particularité de sécréter son propre insecticide (en réalité, une protéine d'origine bactérienne, couramment utilisée en agriculture biologique) ne présente pas de risques nutritionnels.»

Le 19 avril 2004, soit quatre jours avant l'offensive médiatique du Crii-Gen, l'Autorité européenne de sécurité des aliments (Efsa) allait dans le même sens que l'Afssa en se déclarant favorable à l'importation du maïs Mon 863 dans l'Union européenne. Comment des spécialistes peuvent-ils arriver à des conclusions aussi dissemblables sur un même dossier ? «Il s'agit de problèmes très complexes qu'il n'est pas facile d'éclaircir du premier coup. Avant de crier s'affoler, il faut d'abord s'assurer que l'on dispose de résultats scientifiques fiables et confirmés», selon Marc Fellous, le président de la CGB, visiblement agacé par le retentissement de cette affaire. Car les choses n'en sont pas restées là. Intriguée par les résultats de l'étude, la CGB a fait appel à deux pathologistes indépendants de renommée internationale, qui ont procédé à un nouvel examen des coupes histologiques des reins présentant des anomalies.

Leur verdict est sans appel : ces lésions, dont le Crii-gen avait fait grand cas, sont «banales et fréquentes dans la plupart des souches de rat utilisées en toxicologie» et ne peuvent être associées à l'ingestion de maïs transgénique, peut-on lire dans un document de la CGB daté du 14 septembre dernier.

Concernant le poids des reins, plus faible chez les rats nourris au Mon 863, la Commission a requis l'avis du professeur André-Laurent Parodi, 
de l'École nationale vétérinaire de Maisons-Alfort. Ce scientifique explique que «les variations de poids constatées entrent dans la fourchette de la variabilité naturelle. Quant aux anomalies histologiques, je confirme qu'il s'agit bien de néphropathie chronique progressive, une affection qui se développe spontanément chez le rat à partir d'un certain âge, dont la prévalence est plus fréquente chez les mâles que chez les femelles et qui est sans rapport avec l'alimentation.»

Compte tenu de ces nouvelles informations, les conclusions de la CGB sur le Mon 863, publiées sur son site Internet, ne sont évidemment plus les mêmes qu'il y a un an et rejoignent maintenant celles de l'Afssa et de l'Efsa.

Mais l'un de ses membres, le biologiste Gilles-Eric Séralini, responsable scientifique du Crii-Gen, maintient ses doutes. «Il y a eu des négociations avec Monsanto pour extraire les anomalies sanguines de la contre-expertise au motif qu'elles ne sont pas proportionnelles aux doses de maïs ingéré, affirme-t-il. Vu le nombre d'anomalies détectées, il aurait fallu demander à Monsanto de refaire l'expérience, ce qui n'a pas été fait au motif que cela coûte trop cher.»

Sur le fond, il estime «normal» que les données métaboliques n'aient pas été prises en compte. «Une différence même significative de la glycémie n'a pas grand sens si le pancréas des rats est normal comme c'était le cas. En fait, nous sommes face à des résultats qui sont difficilement interprétables et le fait de recommencer l'expérience ne nous aurait pas mieux renseignés. Tout simplement parce que ce type d'essai, conçu à l'origine pour tester des poisons, a un pouvoir discriminant très faible sur des aliments qui ne sont pas censés en être. Depuis cinq ans, des recherches ont été lancées, notamment avec l'appui de l'Europe, pour mettre au point des méthodologies plus sensibles.» Détectera-t-on alors des effets discrets qui échappent pour le moment à l'analyse ? Pour l'heure, les États membres doivent décider à Bruxelles d'autoriser ou non l'importation dans l'Union européenne de ce maïs transgénique qui, jusqu'à preuve du contraire, n'a plus grand-chose d'«inquiétant». Voilà comment de façon inquiétante l'on a toujours alarmé les populations sur les problèmes concernant les OGM.

Les opinions sur les organismes génétiquement modifiés ne doivent pas être un frein à la maîtrise de cette technologie qui quel que soit le bord auquel on appartient est sûrement une solution pour résoudre certains maux dont souffre l'agriculture en Afrique et en Côte d'Ivoire, en particulier.

En Afrique, par exemple, un domaine d'application du génie génétique (cultures in vitro, réalisation de plantes transgéniques) en agriculture est l'amélioration des tubercules et des racines vivrières comme la pomme de terre, la patate douce et le manioc. Cette amélioration permet ainsi 
de maîtriser des maladies et des parasites virulents et d'augmenter la productivité.

La plupart des pays d'Afrique ont un important retard dans le domaine des plantes transgéniques et ont des accès limités à la technologie mis à part l'Egypte, le Sénégal et l'Afrique du Sud. Dans tous ces pays, le problème principal de l'utilisation de ces techniques est l'absence de lois sur le transfert des technologies génétiques. Au Kenya, ce transfert s'effectue dans le cadre d'un partenariat avec les Etats industriels des pays développés.

Au Sénégal, un partenariat avec un laboratoire de recherche universitaire français a permis le développement d'un laboratoire de biotechnologies. Ce partenariat a porté d'une part, sur les travaux de recherche sur l'amélioration de la tolérance au manque d'eau chez une légumineuse originaire d'Afrique (le niébé) et d'autre part, à la formation des spécialistes sénégalais aux biotechnologies.

En Amérique du sud, l'Argentine, le Brésil et le Chili se sont lancés dans les biotechnologies à différents niveaux.

Pour tous ceux que nous avons sondés, tous sont unanimes sur ce constat, l'agriculture ivoirienne doit être systématiquement mécanisée. Car elle utilise des moyens rudimentaires de production et bénéficie de très peu de crédits financiers disponibles pour inciter les jeunes à retourner à la terre.

\section{Conclusion}

L'enquête menée au sein des populations paysannes et intellectuelles a indiqué que très peu de paysans, un peu plus de $65 \%$, n’ont aucune information en ce qui concerne les plantes génétiquement modifiées. Ce fort taux d'ignorance est lié à leur niveau d'instruction.

Par contre, les intellectuels qui se recrutent au niveau de l’Université, des Grandes Écoles et des fonctionnaires des ministères en charge de l'agriculture, de la recherche scientifique, de l'environnement et des étudiants, plus de $97 \%$ connaissent l'existence des OGM.

Au sein des populations paysannes et intellectuelles, il ya à la fois des adversaires et des partisans pour l'éventuel introduction des plantes transgéniques en Côte d'Ivoire.

Les partisans des OGM ont avancé des raisons économiques telles l'amélioration des rendements et la réduction des temps de travail au champ pour se justifier tandis que ceux qui s'y opposent parlent plutôt de danger réel que ces plantes pourraient constituer pour la santé humaine et pour l'environnement et ils évoquent aussi un problème d'éthique morale que les partisans des OGM ignorent.

Entre temps, les paysans favorables aux OGM, contrairement aux plantes transgéniques déjà utilisées dans le monde, préfèrent plutôt des plantes génétiquement modifiées qui résistent au stress hydrique. 
Dans l'ensemble, les ivoiriens reçoivent leurs informations sur les OGM à travers les médias telles que la radio et la télévision y compris tous ce qui y sont farouchement opposés.

La gestion sociale de l'innovation, avec un déficit d'information, une communication non équilibrée, des faits souvent déformés par les médias et les associations opposées aux OGM, des choix politiques pas toujours clairs, et la perte de confiance dans certains acteurs (chercheurs, agriculteurs, agroindustriels et décideurs politiques) ont fini par rendre sceptique un très grand nombre d'individus que nous avons rencontrés en Côte d'Ivoire et en France.

\section{References:}

1. De Cheveigné S., Boy D., Galloux J.C., 2002. Les biotechnologies en débats : pour une démonstration scientifique ed. Balland, Paris $214 \mathrm{p}$.

2. Doré C., Varoquaux F., 2006. Histoire et amélioration de cinquante plantes cultivées. Editions Quae, 812 p.

3. FAO, 2005. La situation mondiale de l'alimentation et de l'agriculture, Trente troisième session, FAO Rome, 19-26 Novembre 2005.

4. FAO, 2002. Evaluation préliminaire de la production céréalière de 2002 en Afrique de l'Ouest. Système d'information et d'alerte rapide (SMIAR), Rapport final, FAO Rome, Décembre 2002.

5. Flowers T.J., M.L. Koyama S.A., Flowers, Chinta sudhakar, K.P. Singh and Yeo A.R., 2002. Their place in engineering tolerance of rice to salinity journal of experimental Botany, vol 51, nunero 342, pp 99-106.

6. Gallais A., Ricroch A. 2006. Plantes transgéniques : faits et enjeux. Synthèses, éditions Quœe, 284 p.

7. Hei Leung. Gene P. Hettel and Ronald P. Cantrelle, 2002. Roles and challenges as we enter the genomics era. International Rice Research Institute, Trends in plant science, 7: 139-142.

8. Heimlich, R. E., Fernandez-Cornejo, J., McBride, W., Klotz-Ingram, C., Jans, S., \& Brooks, N., 2000. Genetically Engineered Crops: Has Adoption Reduced Pesticide Use? Agricultural Outlook: 13-17.

9. Jaglo-Ottosen K., Gilmour S., Zarka D., Schabengerger., ThomasShow M., 1998. Arabidiopsis CBF1 overexpression induces COR genes and enchanges free-zing tolerance. Science 280 : 104-106.

10. Le Déaut J.Y., 2005. Rapport sur la place des biotechnologies en France et en Europe. Rapport du Sénat $n^{\circ} 158$ et de L’Assemblée nationale 2946, Janvier 2005. 40p.

11. Lemarié S. 2000. Analyse économique du développement des cultures à base d'organisme génétiquement modifiées aux Etats unis. 
I. Le développement des OGM agronomiques. Inra-SERD, Grenoble, 42p.

12. Lepoivre P. 2002. Science, décisions et démocratie : l'imbroglio des organismes génétiquement modifiés, Conférence, Faculté universitaire des Sciences agronomiques de Gembloux (www.fsagex.ac.be.//pp//vulgarisation/prudence.htm).

13. Lin W., Price G.K., Fernandez-Cornejo J. 2001. Estimating Farmlevel Effects of Adopting Herbicide Tolerant soybeans. Oil Crops Situation and outlook (USDA-ERS) : 25-34.

14. Mara M.C., 2001. The farm level impacts of transgenic crops: a critical review of the evidence. In Agricultural Biotechnology: Markets and policies in International Setting. IFPRI, Washington, 16p.

15. Morin, 2002 Un riz basmati transgénique résiste à la sécheresse et à l'eau saumâtre http://www.terresacree.org/basmati.htm

16. Oury J.P. 2006. La querelle des OGM, Presse Universitaire de France, $304 \mathrm{p}$.

17. Saito, 2000. Tendances en matière et en sécurité alimentaire dans le monde: une vue d'ensemble, FAO, Rapport de synthèse, 140p.

18. Verfaillie H., 2001. Presentation de Monsento. Goldman Sachs Group Fifth Annual Ag-chimichals AgBiotechnologie Conference, $15 p$.

19. Woodburn A., 2000. Glyphosate: production, pricing and use worldwide. Pest Management Science, 56: 309-312. www.libertyindia.org/policy reports/cotton info march 2002.htm. 